\title{
Influencia del Género en la Percepción y Adopción de e-Learning: Estudio Exploratorio en una Universidad Chilena
}

\author{
Patricio Ramírez-Correa', Francisco Javier Rondán-Cataluña, Jorge Arenas-Gaitán²
}

\begin{abstract}
Based on the Technology Acceptance Model (TAM), the objective of this study is to explore gender differences in adopting e-learning technology for university students in Chile. A multigroup analysis, based on Partial Least Squares (PLS), was used to compare the differences between women and men. The results indicate that in a sample of students of a Chilean University, the conduct of technology acceptance of e-learning, firstly, coincides with the TAM model, and secondly, it isn't statistically significant difference between women and men.
\end{abstract}

Key words: technology acceptance model; gender analysis; e-learning; higher education; Chile.

\section{Resumen}

Basado en el Modelo de Aceptación Tecnológica (TAM), el objetivo del presente estudio es explorar las diferencias de género en la adopción de la tecnología e-learning para los estudiantes universitarios in Chile. Un análisis multigrupo, basado en Partial Least Squares (PLS), se utilizó para comparar las diferencias entre mujeres y hombres. Los resultados indican que en una muestra de estudiantes de una universidad chilena, la conducta de aceptación de la tecnología de elearning, primero, coincide con el modelo TAM, y en segundo lugar, no se observaron diferencias estadísticamente significativas entre mujeres $y$ hombres.

Palabras Claves: modelo de aceptación de tecnología; análisis de género; e-learning; educación superior; Chile.

\footnotetext{
' Departamento de Ingeniería de Sistemas y Computación, Universidad Católica del Norte, Avenida Angamos 0610, Antofagasta, Chile, Tel.: +5655 355167, Email: patricio.ramirez@ucn.cl

${ }^{2}$ Departamento de Administración de Empresas y Comercialización e Investigación de Mercados (Marketing), Universidad de Sevilla, Avenida Ramón y Cajal I, CP 4I018, Sevilla, España, Tel.I: +3495 4554427. Email: rondan@us.es, jarenas@us.es
} 


\section{Introducción}

En el contexto mundial, la Universidad está dentro de un profundo proceso de cambio y el uso de las tecnologías de la información es uno de sus antecedentes significativos. En particular, la adopción de e-learning por estudiantes universitarios está creciendo en el mundo. E-learning, también conocido como aprendizaje basado en web, se define como un proceso de aprendizaje habilitado por Internet (Gunasekaran, McNeil y Saúl, 2002). Si bien en la mayoría de las instituciones universitarias los programas totalmente en web son menos del $5 \%$ de la matrícula total, el número de estudiantes matriculados en al menos un curso con una presencia relevante en línea sería del 30\% al $50 \%$ de la matrícula total (OCDE, 2005). De hecho, el crecimiento real de e-learning en la educación superior es para complementar la instrucción tradicional, y no en los cursos puramente a distancia (Harris, Yanosky y Zastrocky, 2003). Los estudiantes y profesores esperan cada vez más que el e-learning mejore la instrucción en el aula. En ese sentido, el e-learning ha sido crucial para convertir a los métodos de aprendizaje más portátiles y flexibles (Zhang y Nunamaker, 2003). Acorde con lo anterior, desde hace varios años distintas instituciones de educación superior Chilenas aprovechan las ventajas de utilizar e-learning (Muñoz, 2005).

Sin embargo, el género de los estudiantes podría derivar en diferentes percepciones $y$ evaluaciones a similares plataformas e-learning, con claras implicancias en el logro de los resultados esperados. El género se considera un eje fundamental para explicar las desigualdades e identidades en la sociedad moderna (Walby, 1990), y en el caso de la adopción de tecnologías de información, la literatura indica al género como clave para entender diferencias en el rol de las percepciones de utilidad y facilidad de uso como determinantes de esta adopción (Ong y Lai, 2006; SánchezFranco, Villarejo-Ramos y Rondan-Cataluña, 2006; Venkatesh y Morris, 2000). Por otra parte, habida cuenta que el propósito y uso común de estas tecnologías puede reducir al mínimo las diferencias asociadas al género ¿Cuál es la influencia del género sobre cómo los estudiantes universitarios adoptan la tecnología de información para proporcionar soluciones de aprendizaje de manera eficiente y eficaz? En ese sentido, Kim y Forsythe (2008) reportan que no existen diferencias estadísticamente significativas entre hombres y mujeres en el proceso de adopción de una particular tecnología web. De acuerdo con estas ideas, existe una clara discusión sobre la influencia de las diferencias de género en adopción de elearning. La importancia de esta discusión está determinada por la necesidad de saber si plataformas e-learning son aceptadas, usadas y percibidas de la misma manera por todos los estudiantes, o si las diferencias de género deben tenerse en cuenta en este proceso.

El objetivo principal de este estudio es explorar las diferencias de género en la adopción de la tecnología en los estudiantes de educación superior en Chile. El modelo TAM es la herramienta utilizada para medir la aceptación y el uso de e-learning de los encuestados. Luego de valorar el modelo TAM en la totalidad de la muestra con Partial Least Squares (PLS), ésta se dividió en dos grupos de estudiantes: mujeres y hombres. Se utilizó ANOVA para comparar la valoración de los constructos, y el análisis PLS de múltiples grupos para comparar las diferencias entre grupos.

\section{Revisión de la Literatura}

\section{E-Learning en la Educación Superior}

E-learning se está convirtiendo en un elemento importante de la educación superior. Los primeros cursos en la web surgieron en 1995 y han tenido una rápida expansión en el contexto mundial, acorde al acelerado aumento del acceso de los estudiantes a Internet. En 1996, Murray Goldberg desarrolló un paquete de software llamado WebCT diseñado para permitir que los cursos basados en web se ofrezcan a través de Internet (Bates, 2005). Luego de WebCT, varios sistemas de aprendizaje basados en páginas web han sido desarrollados para los colegios $y$ universidades (Ejemplos: WebCH, Blackboard, Claroline y Smile).

El aprendizaje basado en web debe tener en cuenta que la educación ha activado un cambio del paradigma de la enseñanza al paradigma del aprendizaje. Como resultado, los estudiantes son cada vez más independientes del profesor. Lamentablemente, gran parte del desarrollo de e-learning se lleva a cabo sin una verdadera comprensión de las cuestiones que son propias de aprendizaje basado en web (Hadjerrouit, 2006). Por ejemplo, las actividades realizadas en Internet se han incorporado a las clases presenciales como un recurso añadido, sin reducir el tiempo de clases (Bates, 2005). 
Para profesores y estudiantes, las implicaciones del elearning son grandes. Cada vez más las universidades deben ofrecer calidad y flexibilidad para satisfacer las diversas necesidades de los estudiantes, lo que inevitablemente implicará adaptar los cursos para satisfacer las diferentes aspiraciones y necesidades educativas. Otra implicación de la enseñanza virtual es el aumento de la competencia internacional entre universidades, los nuevos métodos de comunicación son herramientas muy útiles para facilitar la internacionalización de la educación superior (O’Neill, Singh y O'Donoghue, 2004). Además, el e-learning refleja una nueva dinámica de respuestas a las necesidades de la sociedad del conocimiento e implica la libertad y la igualdad de acceso al conocimiento más allá de las fronteras culturales y sociales (Raza y Murad, 2008).

\section{E-Learning y el Modelo TAM}

Propuesto por Fred Davis (David, 1989), el modelo de aceptación de la tecnología (TAM) explica el proceso de aceptación de la tecnología de la información a nivel individual. TAM postula que la intención de conducta individual de uso de la tecnología de la información queda determinada por la utilidad percibida y la facilidad de uso percibida. Al mismo tiempo, la facilidad de uso percibida afecta directamente la utilidad percibida. Venkatesh y Bala (2008) proponen un modelo denominado TAM 3 que amplia TAM con un conjunto de factores predictores $y$ moderadores. .

En la literatura existen diversos estudios que utilizan TAM para evaluar la aceptación del usuario de e-learning en estudiantes universitarios, profesores y trabajadores. La mayoría de estos estudios amplían el modelo TAM mediante factores predictores y moderadores, tales como: las normas subjetivas (Grandon, Alshare, y Kwun, 2005; Lee, Cho, Gay, Davidson y Ingraffea, 2003; Park, 2009; Van Raaij y Schepers, 2008; Yuen y Ma , 2008); auto-eficacia computacional (Chang y Tung, 2008; Grandon et al., 2005; Hayashi, Chen, Ryan y Wu, 2004; Ong y Lai, 2006; Ong, Lai y Wang, 2004; Park, 2009; Yuen y Ma, 2008); disfrute percibido (Chen, Chen, Lin y Yeh, 2007; Roca y Gagné, 2008; Zhang, Zhao y Tan, 2008); absorción cognitiva (Liu, Liao, y Pratt, 2009; Saade y Bahli, 2005); características del sistema (Chang y Tung, 2008; Chen et al., 2007; Liu et al., 2009; Park, 2009); ansiedad computacional (Van Raaij y Schepers, 2008); género (Ong y Lai, 2006); factores motivacionales (Park, Lee y Cheong, 2007; Roca y Gagné,
2008); capacidad de innovación personal (Van Raaij y Schepers, 2008); apoyo técnico (Ngai, Poon y Chan, 2007); credibilidad percibida (Ong et al., 2004); y compatibilidad (Chang y Tung, 2008). Algunos resultados relevantes para este estudio se presentan a continuación.

El modelo TAM se ha utilizado con éxito en el contexto de e-learning (Roca, Chiu y Martínez, 2006). Saadé, Nebebe y Tan (2007) indican que TAM se puede extender a la multimedia y e-contexto de aprendizaje. Relacionado a lo anterior, Liu et al. (2009) confirmaron que la riqueza de los medios de comunicación es una variable externa que influye en la intención de comportamiento de los estudiantes universitarios de utilizar e-learning. En particular, Halawi y McCarthy (2008) revelaron que los estudiantes utilizan e-learning, si es útil y fácil de usar. Hayashi et al. (2004) verificó que la utilidad percibida afecta directamente a la intención del alumno para continuar utilizando e-learning. Asimismo, Ngai et al. (2007) especifican que la facilidad de uso percibida y la utilidad percibida son los principales factores que afectan a la actitud de los estudiantes universitarios para utilizar elearning.

Como en otros contextos, los elementos subjetivos son de gran importancia en el momento de adoptar e-learning. Lee et al. (2003) demuestran que la expectativa inicial del estudiante afecta la percepción de las actitudes hacia el sistema y el uso del sistema. Además, el cambio de actitud del estudiante es significativamente influenciado por los cambios de actitud de otros estudiantes. Saade y Bahli (2005) indican que la absorción cognitiva (definido como un estado de profunda implicación con el sistema) influye en la utilidad percibida y en la facilidad de uso percibida. Asociado a ello, Liu et al. (2009) confirmaron que el nivel de concentración se correlaciona positivamente con la intención de utilizar e-learning. Por otra parte, Park et al. (2007) señalan que la motivación es un factor que influye en la adopción y uso del e-learning por los instructores.

Otro elemento importante para la adopción de e-learning es el disfrute percibido en su uso, definido éste como el grado en que la actividad de usar una tecnología de la información se percibe como personalmente agradable por sí misma, aparte de el valor instrumental de la tecnología. Roca y Gagné (2008) indican que el disfrute percibido afecta directamente tanto a la utilidad percibida como a la facilidad de uso percibida. Además el disfrute percibido 
afecta directamente a la intención de uso. Consistentemente, Chen et al. (2007) muestran que el disfrute percibido afecta significativamente la percepción de los estudiantes sobre la utilidad y Zhang et al. (2008) señalan que el disfrute percibido tiene un efecto positivo sobre la intención del comportamiento de los estudiantes universitarios a utilizar e-learning.

Finalmente, existen evidencias sobre diferencias asociadas al género en la adopción de e-learning en trabajadores. Ong y Lai (2006) muestran que las puntuaciones de los hombres acerca de la utilidad percibida, la facilidad de uso percibida y la intención de comportamiento para el uso de e-learning son más altas que las puntuaciones de las mujeres. Además, la utilidad percibida influye en la intención de comportamiento para el uso de e-learning con más fuerza para los hombres que para las mujeres $y$, del mismo modo, la facilidad de uso percibida influye en la utilidad percibida del e-learning con más fuerza en las mujeres que en los hombres.

\section{Modelo Propuesto e Hipótesis}

\section{Modelo}

El modelo TAM propuesto se muestra en la Figura I.

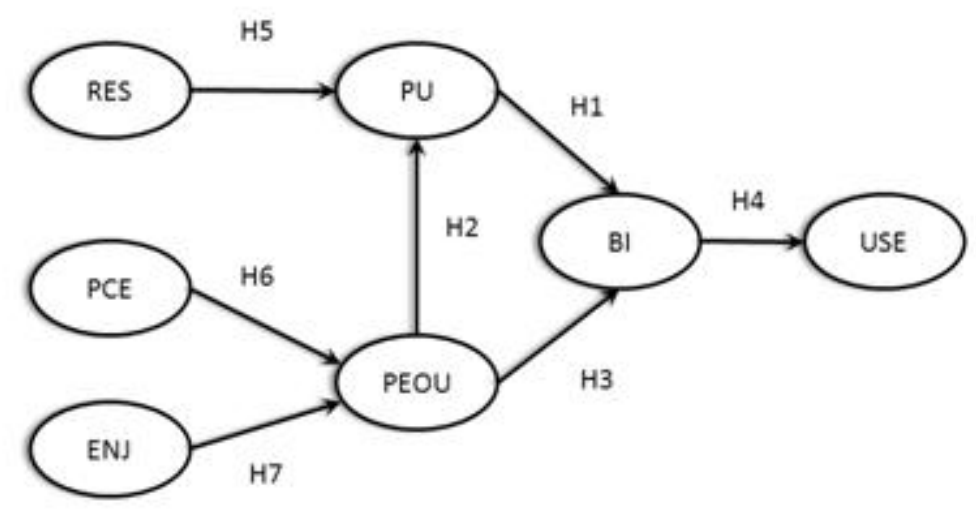

Figura I. Modelo Propuesto

En el modelo son propuestas relaciones entre Utilidad Percibida (PU), Facilidad de Uso Percibida (PEOU) y Intención de Comportamiento $(\mathrm{BI})$, además se incluye el efecto de $\mathrm{BI}$ sobre Uso de la Plataforma e-Learning (USE). Este modelo TAM básico se enriquece con tres antecedentes, por una parte, Demostrabilidad del Resultado (RES) antecede a PU, y por otra, Percepción de Control Externo (PCE) y Disfrute Percibido (ENJ) anteceden a PEOU.

\section{Hipótesis}

Basados en Halawi y McCarthy (2008) y Venkatesh y Morris (2000) se proponen las siguientes hipótesis:
- HI: PU se relaciona positivamente con $\mathrm{BI}$ en la adopción de e-learning en la educación superior.

- H2: PEOU se relaciona positivamente con PU en la adopción de e-learning en la educación superior.

- H3: PEOU se relaciona positivamente con $\mathrm{BI}$ en la adopción de e-learning en la educación superior.

- H4: BI se relaciona positivamente con USE en la adopción de e-learning en la educación superior. 
Basados en Chang y Tung (2008), Chen et al. (2007), Roca y Gagné (2008), Venkatesh y Bala (2008) y Zhang et al. (2008) se proponen las siguientes hipótesis:

- H5: RES se relaciona positivamente con PU en la adopción de e-learning en la educación superior.

- H6: PCE se relaciona positivamente con PEOU en la adopción de e-learning en la educación superior.

- H7: ENJ se relaciona positivamente con PEOU en la adopción de e-learning en la educación superior.

Basados en Ong y Lai (2006) se proponen las siguientes hipótesis:

- H8a: Existen diferencias estadísticamente significativas entre hombres $y$ mujeres en las puntuaciones de variables de adopción de e-learning en la educación superior.

- H8b: Existen diferencias estadísticamente significativas entre hombres y mujeres en relaciones entre variables de la adopción de e-learning en la educación superior.

\section{Método de Investigación}

\section{Muestra}

La investigación empírica se basó en un muestreo no aleatorio de conveniencia. En particular, los datos fueron recolectados en Chile a través de un cuestionario en línea entre mayo y julio de 2009. El cuestionario en línea fue enviado a los estudiantes de las carreras de ingeniería industrial e ingeniería informática de la Universidad Católica del Norte (Chile) que usan su plataforma elearning Claroline. La exclusión de los cuestionarios inválidos debido a duplicaciones o campos vacíos proporcionó un tamaño final de la muestra de 159 estudiantes, 116 hombres $(73 \%)$ y 43 mujeres (27\%). La composición de género de esta muestra es acorde con el universo explorado (un $79 \%$ de estudiantes hombre y un $21 \%$ de estudiantes mujeres). La edad media de los encuestados es de 22 años y han estado estudiando durante 4 años (en promedio) en la Universidad.

\section{Escalas}

Las escalas de medición aplicados han sido ampliamente probadas en otras investigaciones. Específicamente, para medir los constructos de TAM se han adaptado las escalas propuestas por Venkatesh y Bala (2008).

\section{Análisis y Resultados}

\section{Estadísticas Descriptivas y ANOVA}

Los resultados de las estadísticas descriptivas son mostrados en la tabla I. La escala utilizada es tipo Likert de 5 puntos, salvo la variable USE que informa minutos semanales. El software SPSS fue usado para este examen.

\begin{tabular}{ccccccccc}
\hline & \multicolumn{2}{c}{ Total $(\mathrm{N}=159)$} & \multicolumn{2}{c}{ Hombres $(\mathrm{N}=1 \mid$ l6) } & \multicolumn{2}{c}{ Mujeres $(\mathrm{N}=43)$} & \multicolumn{2}{c}{ ANOVA } \\
\cline { 2 - 8 } Variable Latente & Promedio & Desv. típica & Promedio & Desv. típica & Promedio & Desv. típica & $\mathrm{F}$ & Sig. \\
\hline BI & 4,0 & 0,84 & 4,0 & 0,86 & 4,2 & 0,76 & 2,04 & 0,155 \\
ENJ & 3,5 & 0,91 & 3,4 & 0,97 & 3,5 & 0,74 & 0,35 & 0,556 \\
PCE & 4,2 & 0,75 & 4,2 & 0,80 & 4,3 & 0,60 & 1,67 & 0,198 \\
PEOU & 4,2 & 0,80 & 4,2 & 0,85 & 4,4 & 0,63 & 2,01 & 0,158 \\
PU & 4,0 & 0,83 & 4,0 & 0,89 & 4,1 & 0,66 & 0,72 & 0,396 \\
RES & 3,8 & 0,87 & 3,7 & 0,91 & 3,9 & 0,72 & 1,91 & 0,169 \\
USE & 44,4 & 27,71 & 43,3 & 28,45 & 47,5 & 25,67 & 0,73 & 0,393 \\
\hline
\end{tabular}

Tabla I. Estadísticas descriptivas y resultado de ANOVA 
Luego de probar la normalidad y homocedasticidad de las variables, y tal como se puede apreciar en las últimas dos columnas de la tabla $\mathrm{I}$, el resultado del test ANOVA no indica diferencias estadísticamente significativas entre las puntuaciones de hombres y mujeres. Por tanto, se refuta la H8a.

\section{Valoración del Modelo de Investigación}

Un enfoque Partial Least Squares (PLS) para Modelos de Ecuaciones Estructurales (SEM) se usó para testar el modelo de investigación (Chin, 1998; Tenenhaus, Vinzi, Chatelin y Lauro, 2005). Inicialmente, se valoró el modelo propuesto para el conjunto de la muestra (159 casos). A continuación la muestra se dividió en dos grupos: hombres, con 116 casos y mujeres, con 43 casos. El análisis multigrupo de PLS se usó para comparar las diferencias entre grupos. El software SmartPLS 2.0 de M3 se utilizó para este análisis (Ringle, Wende y Will, 2005).
Un modelo PLS es descrito por dos modelos: (I) un modelo de medida entre las variables manifiestas (VMs) y sus propias variables latentes (VLs) y (2) un modelo estructural entre algunas variables endógenas a otras variables.

\section{Análisis del Modelo de Medida}

Previo a analizar el modelo estructural, la fiabilidad y validez de los modelos de medida fueron determinadas. La fiabilidad individual se evaluó examinado las cargas $(\lambda) \circ$ correlaciones simples de las medidas o indicadores con sus respectivas $\mathrm{VL}$ (se aceptaron los indicadores con $\lambda \geq$ $0,707)$. En las tablas 2,3 y 4 se muestran los resultados del procedimiento de carga cruzada de toda la muestra, grupo hombres y grupo mujeres, respectivamente.

\begin{tabular}{cccccccc}
\hline & BI & ENJ & PCE & PEOU & PU & RES & USE \\
BII & 0,80 & 0,40 & 0,47 & 0,30 & 0,39 & 0,34 & 0,20 \\
BI2 & 0,90 & 0,43 & 0,41 & 0,41 & 0,44 & 0,45 & 0,23 \\
BI3 & 0,84 & 0,31 & 0,30 & 0,47 & 0,40 & 0,39 & 0,32 \\
ENJI & 0,45 & 0,87 & 0,12 & 0,23 & 0,39 & 0,46 & 0,12 \\
ENJ2 & 0,38 & 0,93 & 0,20 & 0,37 & 0,43 & 0,40 & - \\
ENJ3 & 0,38 & 0,87 & 0,06 & 0,24 & 0,30 & 0,43 & 0,02 \\
PCEI & 0,40 & 0,09 & 0,82 & 0,49 & 0,38 & 0,13 & 0,17 \\
PCE2 & 0,38 & 0,18 & 0,80 & 0,43 & 0,42 & 0,16 & 0,11 \\
PCE3 & 0,35 & 0,13 & 0,86 & 0,59 & 0,40 & 0,12 & 0,03 \\
PEOUI & 0,49 & 0,34 & 0,58 & 0,89 & 0,57 & 0,26 & 0,21 \\
PEOU2 & 0,37 & 0,22 & 0,57 & 0,87 & 0,48 & 0,15 & 0,17 \\
PEOU3 & 0,41 & 0,28 & 0,51 & 0,89 & 0,45 & 0,26 & 0,24 \\
PEOU4 & 0,30 & 0,27 & 0,42 & 0,78 & 0,31 & 0,18 & 0,17 \\
PUI & 0,40 & 0,41 & 0,46 & 0,43 & 0,88 & 0,42 & 0,15 \\
PU2 & 0,43 & 0,34 & 0,44 & 0,48 & 0,87 & 0,30 & 0,20 \\
PU3 & 0,36 & 0,37 & 0,37 & 0,46 & 0,88 & 0,31 & 0,16 \\
PU4 & 0,49 & 0,37 & 0,41 & 0,51 & 0,86 & 0,34 & 0,14 \\
RESI & 0,44 & 0,38 & 0,14 & 0,23 & 0,26 & 0,85 & 0,09 \\
RES2 & 0,43 & 0,40 & 0,18 & 0,26 & 0,34 & 0,88 & 0,15 \\
RES3 & 0,36 & 0,44 & 0,11 & 0,18 & 0,40 & 0,88 & 0,07 \\
USE & 0,30 & 0,04 & 0,12 & 0,23 & 0,19 & 0,12 & 1,00 \\
\hline
\end{tabular}

Tabla 2. Resultados del procedimiento de carga cruzada de toda la muestra 


\begin{tabular}{cccccccc}
\hline & BI & ENJ & PCE & PEOU & PU & RES & USE \\
BII & 0,78 & 0,44 & 0,46 & 0,29 & 0,40 & 0,31 & 0,13 \\
BI2 & 0,90 & 0,50 & 0,43 & 0,43 & 0,46 & 0,48 & 0,17 \\
BI3 & 0,86 & 0,41 & 0,29 & 0,48 & 0,40 & 0,38 & 0,33 \\
ENJI & 0,52 & 0,89 & 0,20 & 0,26 & 0,43 & 0,56 & 0,10 \\
ENJ2 & 0,44 & 0,92 & 0,22 & 0,37 & 0,48 & 0,48 & 0,00 \\
ENJ3 & 0,48 & 0,90 & 0,09 & 0,31 & 0,33 & 0,51 & 0,03 \\
PCEI & 0,39 & 0,12 & 0,83 & 0,49 & 0,45 & 0,08 & 0,16 \\
PCE2 & 0,40 & 0,18 & 0,82 & 0,49 & 0,48 & 0,15 & 0,11 \\
PCE3 & 0,35 & 0,17 & 0,87 & 0,64 & 0,46 & 0,10 & 0,00 \\
PEOUI & 0,55 & 0,39 & 0,64 & 0,89 & 0,65 & 0,28 & 0,26 \\
PEOU2 & 0,40 & 0,24 & 0,59 & 0,89 & 0,50 & 0,14 & 0,21 \\
PEOU3 & 0,40 & 0,33 & 0,57 & 0,91 & 0,51 & 0,21 & 0,27 \\
PEOU4 & 0,30 & 0,25 & 0,44 & 0,80 & 0,38 & 0,20 & 0,22 \\
PUI & 0,38 & 0,45 & 0,53 & 0,50 & 0,89 & 0,40 & 0,16 \\
PU2 & 0,47 & 0,40 & 0,50 & 0,55 & 0,90 & 0,31 & 0,28 \\
PU3 & 0,41 & 0,38 & 0,47 & 0,51 & 0,90 & 0,32 & 0,18 \\
PU4 & 0,49 & 0,40 & 0,46 & 0,56 & 0,85 & 0,33 & 0,16 \\
RESI & 0,44 & 0,47 & 0,08 & 0,20 & 0,26 & 0,86 & 0,06 \\
RES2 & 0,44 & 0,50 & 0,15 & 0,26 & 0,35 & 0,89 & 0,15 \\
RES3 & 0,35 & 0,50 & 0,10 & 0,18 & 0,37 & 0,87 & 0,07 \\
USE & 0,26 & 0,04 & 0,10 & 0,27 & 0,22 & 0,11 & 1,00 \\
\hline
\end{tabular}

Tabla 3. Resultados del procedimiento de carga cruzada de grupo hombres

\begin{tabular}{cccccccc}
\hline & BI & ENJ & PCE & PEOU & PU & RES & USE \\
BII & 0,85 & 0,14 & 0,48 & 0,30 & 0,33 & 0,38 & 0,44 \\
BI2 & 0,92 & 0,16 & 0,35 & 0,24 & 0,39 & 0,28 & 0,41 \\
BI3 & 0,79 & 0,17 & 0,36 & 0,39 & 0,42 & 0,39 & 0,26 \\
ENJI & 0,21 & 0,71 & $-0,22$ & 0,08 & 0,26 & 0,06 & 0,21 \\
ENJ2 & 0,13 & 0,96 & 0,11 & 0,33 & 0,21 & 0,06 & $-0,06$ \\
ENJ3 & 0,00 & 0,70 & $-0,21$ & $-0,11$ & 0,15 & 0,06 & $-0,06$ \\
PCEI & 0,43 & 0,21 & 0,89 & 0,50 & 0,13 & 0,31 & 0,21 \\
PCE2 & 0,27 & 0,05 & 0,70 & 0,07 & 0,11 & 0,13 & 0,11 \\
PCE3 & 0,32 & $-0,05$ & 0,73 & 0,32 & 0,07 & 0,17 & 0,11 \\
PEOUI & 0,23 & 0,29 & 0,39 & 0,84 & 0,14 & 0,14 & 0,00 \\
PEOU2 & 0,24 & 0,33 & 0,52 & 0,79 & 0,37 & 0,16 & 0,00 \\
PEOU3 & 0,40 & 0,18 & 0,31 & 0,80 & 0,20 & 0,38 & 0,11 \\
PEOU4 & 0,28 & 0,45 & 0,31 & 0,72 & 0,01 & 0,10 & 0,02 \\
PUI & 0,45 & 0,18 & 0,15 & 0,14 & 0,88 & 0,50 & 0,10 \\
PU2 & 0,25 & $-0,02$ & 0,10 & 0,12 & 0,70 & 0,24 & $-0,14$ \\
PU3 & 0,21 & 0,26 & $-0,07$ & 0,24 & 0,77 & 0,27 & 0,11 \\
PU4 & 0,44 & 0,29 & 0,17 & 0,30 & 0,89 & 0,38 & 0,06 \\
RESI & 0,39 & 0,00 & 0,47 & 0,32 & 0,26 & 0,79 & 0,20 \\
RES2 & 0,38 & $-0,02$ & 0,27 & 0,22 & 0,28 & 0,87 & 0,14 \\
RES3 & 0,34 & 0,11 & 0,15 & 0,15 & 0,52 & 0,92 & 0,05 \\
USE & 0,44 & 0,01 & 0,21 & 0,04 & 0,06 & 0,13 & 1,00 \\
\hline
\end{tabular}

Tabla 4. Resultados del procedimiento de carga cruzada de grupo mujeres 
La fiabilidad de la VL indica la rigurosidad con que las variables observadas miden la misma VL, El coeficiente $\alpha$ de Cronbach fue utilizado como el índice de fiabilidad de las VLs (fueron aceptadas VLs con $\alpha>0,7$ ). Además, fiabilidad compuesta fue calculada. La validez convergente de la VL se evaluó mediante el examen de la varianza media extraída (AVE), véase Fornell y Larcker (I98I) (se aceptaron AVE> 0,5). La Tabla 5 muestra el coeficiente $\alpha$ de Cronbach, AVE y fiabilidad compuesta por cada VL.

\begin{tabular}{crrrrrrrrrr}
\hline & \multicolumn{3}{c}{ Todos } & \multicolumn{4}{c}{ Hombres } & \multicolumn{3}{c}{ Mujeres } \\
\cline { 2 - 10 } & $\begin{array}{c}\alpha \text { de } \\
\text { Cronbach }\end{array}$ & AVE & $\begin{array}{c}\text { Fiabilidad } \\
\text { compuesta }\end{array}$ & $\begin{array}{c}\alpha \text { de } \\
\text { Cronbach }\end{array}$ & AVE & $\begin{array}{c}\text { Fiabilidad } \\
\text { compuesta }\end{array}$ & $\begin{array}{c}\alpha \text { de } \\
\text { Cronbach }\end{array}$ & AVE & $\begin{array}{c}\text { Fiabilidad } \\
\text { compuesta }\end{array}$ \\
\hline BI & 0,8 & 0,72 & 0,89 & 0,8 & 0,72 & 0,89 & 0,8 & 0,73 & 0,89 \\
ENJ & 0,9 & 0,80 & 0,92 & 0,9 & 0,81 & 0,93 & 0,8 & 0,54 & 0,76 \\
PCE & 0,8 & 0,68 & 0,86 & 0,8 & 0,70 & 0,88 & 0,7 & 0,54 & 0,77 \\
PEOU & 0,9 & 0,74 & 0,92 & 0,9 & 0,76 & 0,93 & 0,8 & 0,62 & 0,87 \\
PU & 0,9 & 0,76 & 0,93 & 0,9 & 0,79 & 0,94 & 0,8 & 0,65 & 0,88 \\
RES & 0,8 & 0,76 & 0,90 & 0,8 & 0,76 & 0,91 & 0,8 & 0,74 & 0,89 \\
USE & 1,0 & 1,00 & 1,00 & 1,0 & 1,00 & 1,00 & 1,0 & 1,00 & 1,00 \\
\hline
\end{tabular}

Tabla 5. Coeficiente $\alpha$ de Cronbach, AVE y fiabilidad compuesta

Se probó la validez discriminante de las VLs al analizando correlaciones con el resto de las LVs (véanse las tablas 6, si la raíz cuadrada del AVE de cada LV es mayor que las 7 y 8 ).

\begin{tabular}{crrrrrrr}
\hline & BI & ENJ & PCE & PEOU & PU & RES & USE \\
BI & 0,85 & - & - & - & - & - & - \\
ENJ & 0,45 & 0,89 & - & - & - & - & - \\
PCE & 0,45 & 0,16 & 0,82 & - & - & - & - \\
PEOU & 0,47 & 0,33 & 0,62 & 0,86 & - & - & - \\
PU & 0,49 & 0,43 & 0,48 & 0,54 & 0,87 & - & - \\
RES & 0,46 & 0,47 & 0,16 & 0,25 & 0,39 & 0,87 & - \\
USE & 0,30 & 0,04 & 0,12 & 0,23 & 0,19 & 0,12 & 1,00 \\
\hline
\end{tabular}

Tabla 6. Correlaciones de VL para toda la muestra (elementos en la diagonal son las raíces cuadradas del AVE)

\begin{tabular}{crrrrrrr}
\hline & BI & ENJ & PCE & PEOU & PU & RES & USE \\
BI & 0,85 & - & - & - & - & - & - \\
ENJ & 0,53 & 0,90 & - & - & - & - & - \\
PCE & 0,45 & 0,19 & 0,84 & - & - & - & - \\
PEOU & 0,49 & 0,35 & 0,65 & 0,87 & - & - & - \\
PU & 0,49 & 0,46 & 0,55 & 0,60 & 0,89 & - & - \\
RES & 0,47 & 0,57 & 0,13 & 0,24 & 0,38 & 0,87 & - \\
USE & 0,26 & 0,04 & 0,10 & 0,27 & 0,22 & 0,11 & 1,00 \\
\hline
\end{tabular}

Tabla 7. Correlaciones de VL para el grupo de hombres (elementos en la diagonal son las raíces cuadradas del AVE) 


\begin{tabular}{crrrrrrr}
\hline & BI & ENJ & PCE & PEOU & PU & RES & USE \\
BI & 0,85 & - & - & - & - & - & - \\
ENJ & 0,18 & 0,73 & - & - & - & - & - \\
PCE & 0,47 & 0,13 & 0,74 & - & - & - & - \\
PEOU & 0,36 & 0,40 & 0,50 & 0,79 & - & - & - \\
PU & 0,45 & 0,23 & 0,13 & 0,25 & 0,81 & - & - \\
RES & 0,41 & 0,05 & 0,30 & 0,24 & 0,45 & 0,86 & - \\
USE & 0,44 & 0,01 & 0,21 & 0,04 & 0,06 & 0,13 & 1,00 \\
\hline
\end{tabular}

Tabla 8. Correlaciones de VL para el grupo de mujeres (elementos en la diagonal son las raíces cuadradas del AVE)

\section{Análisis del Modelo Estructural}

Después de comprobar la validez y la fiabilidad del modelo de medida, las relaciones entre los constructos fueron probadas. Las hipótesis fueron evaluadas mediante el examen de los coeficientes de camino $(\beta)$ y sus niveles de significación (se aceptaron $\beta>=0,2$ ). Un bootstrapping con
500 sub-muestras fue realizado para comprobar la significación estadística de cada uno de los coeficientes de los caminos. La varianza explicada (R-cuadrado) en las VLs endógenas y el coeficiente de significación de regresión ( $F$ test), sirven como indicadores de la capacidad explicativa del modelo. La figura 2 muestra el resultado del análisis PLS con toda la muestra.

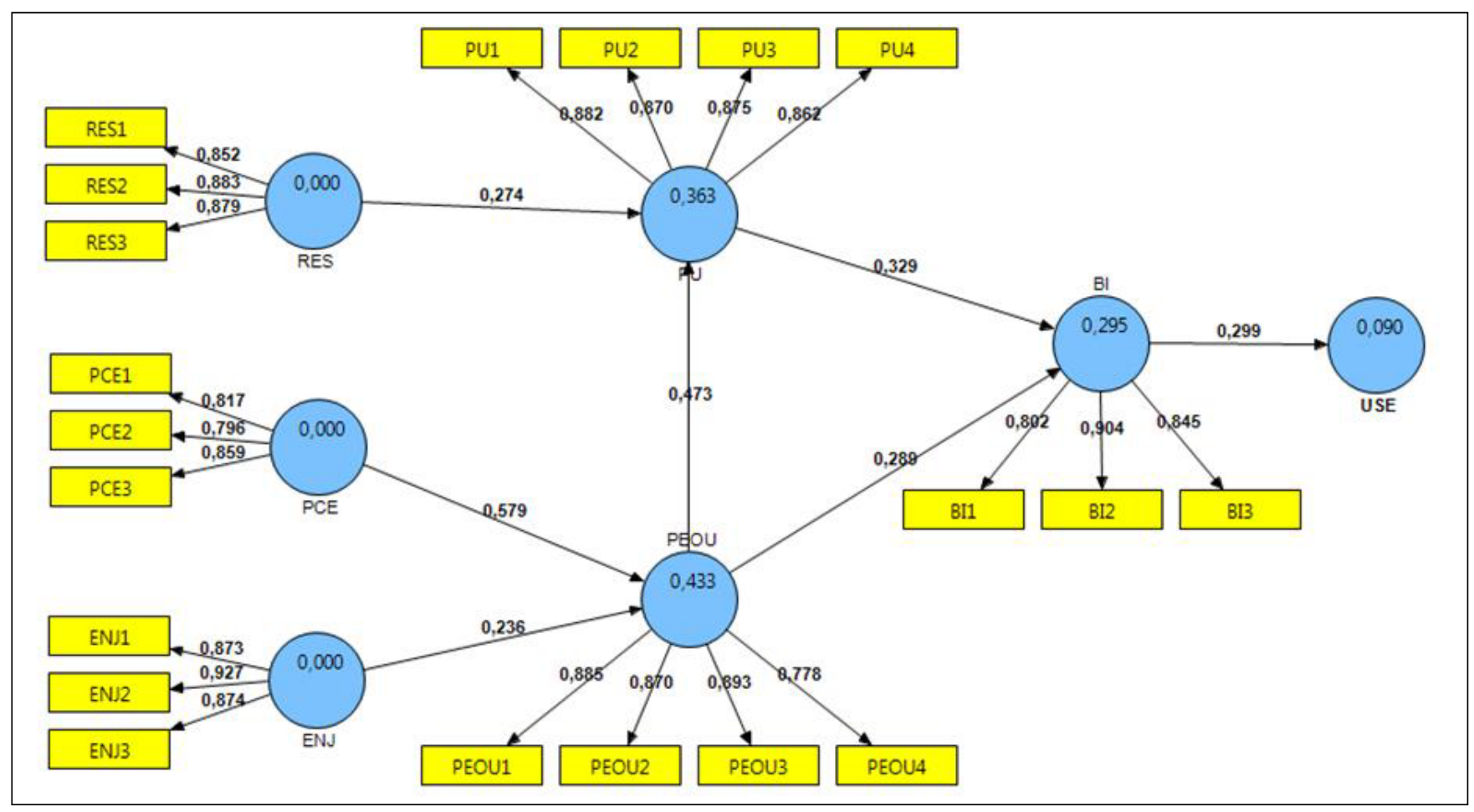

Figura 2. Modelo Todos: R-cuadrado y camino estimado del análisis PLS 
Los resultados de los análisis PLS para el modelo con toda la muestra, el grupo de los hombres y el grupo de las mujeres se muestran en las tablas 9 y 10 . Con base a estos resultados, se aceptan las hipótesis $\mathrm{HI}, \mathrm{H} 2, \mathrm{H} 3, \mathrm{H} 4$, $\mathrm{H} 5, \mathrm{H} 6$ y $\mathrm{H} 7$.

\begin{tabular}{crrr}
\hline Variable Endógena & \multicolumn{1}{l}{ Todos } & Hombres & Mujeres \\
\hline BI & 0,29 & 0,30 & 0,27 \\
PEOU & 0,43 & 0,48 & 0,36 \\
PU & 0,36 & 0,42 & 0,22 \\
USE & 0,09 & 0,07 & 0,19 \\
\hline
\end{tabular}

Tabla 9. Varianza explicada (R-cuadrado) en las VLs endógenas.

\begin{tabular}{|c|c|c|c|c|c|c|c|c|}
\hline Camino & Todos & (Sig.) & Hombres & (Sig.) & Mujeres & (Sig.) & t-spooled & (Sig.) \\
\hline \multirow{2}{*}{$\mathrm{BI}$-> USE } & & $* * *$ & & $* *$ & & $* * *$ & $-0,409947$ & n.s. \\
\hline & 0,30 & & 0,26 & & 0,44 & & & \\
\hline \multirow[t]{2}{*}{ ENJ -> PEOU } & & $*$ & & $* *$ & & n.s. & $-0,197777$ & n.s. \\
\hline & 0,24 & & 0,24 & & 0,34 & & & \\
\hline \multirow[t]{2}{*}{ PCE -> PEOU } & & **** & & $* * *$ & & $* * *$ & $0,3254 \mid 87$ & n.s. \\
\hline & 0,58 & & 0,61 & & 0,46 & & & \\
\hline \multirow[t]{2}{*}{ PEOU -> BI } & & $*$ & & * & & * & 0,0401864 & n.s. \\
\hline & 0,29 & & 0,29 & & 0,27 & & & \\
\hline \multirow[t]{2}{*}{ PEOU -> PU } & & $* * *$ & & $* * *$ & & n.s. & 0,8193262 & n.s. \\
\hline & 0,47 & & 0,54 & & 0,15 & & & \\
\hline \multirow[t]{2}{*}{ PU $->\mathrm{BI}$} & & $*$ & & * & & $* * *$ & $-0,093988$ & n.s. \\
\hline & 0,33 & & 0,32 & & 0,38 & & & \\
\hline \multirow[t]{2}{*}{ RES -> PU } & & $* *$ & & $* *$ & & $* * *$ & $-0,36554$ & n.s. \\
\hline & 0,27 & & 0,25 & & 0,42 & & & \\
\hline
\end{tabular}

Tabla 10. Coeficientes de camino $(\beta)$ y comparación entre grupos (Sig. * $p=0,05 * * p=0,01 * * * p=0,001$ )

Con el propósito de comparar los modelos estructurales del grupo hombres y del grupo mujeres, se realizo un análisis PLS de múltiples grupos (Chin, 2000; Keil, Tan, Wei, Saarinen, Tuunainen y Wassenaar, 2000). Las últimas dos columnas de la tabla 10 indican este análisis, su resultado permite refutar la hipótesis $\mathrm{H} 8 \mathrm{~b}$.

\section{Conclusión}

En conclusión queremos destacar cuatro contribuciones de este estudio exploratorio. Primero, se ha podido utilizar exitosamente el modelo TAM ampliado con otros constructos utilizados en TAM3 para explicar el proceso de adopción de e-learning en la educación superior en chilena. Segundo, un hallazgo importante es la comprobación de una fuerte y significativa relación entre
Percepción de Control Externo y Percepción de Facilidad de Uso de la plataforma e-learning, lo que tiene implicaciones para el diseño de estas plataformas en relación al control y recursos otorgados a los usuarios. Tercero, acorde a la literatura anterior, fue posible encontrar una relación significativa entre el Disfrute Percibido y la Percepción de Facilidad de Uso, como entre Demostrabilidad del Resultado y Utilidad Percibida. Cuarto y último, el estudio indica que no existen diferencias estadísticamente significativas entre estudiantes universitarios hombres y mujeres de la muestra al momento de adoptar una plataforma e-learning. Si bien los resultados de Ong y Lai (2006) señalan diferencias relacionadas al género en la adopción de plataformas elearning, nuestros resultados están alineados con el trabajo de Kim y Forsythe (2008). Opinamos que la aplicación en 
una muestra de jóvenes universitarios (y no de trabajadores), es clave para explicar este contraste de resultados. Es muy posible que en este tipo de población exista una mayor homogeneidad respecto a las nuevas tecnologías porque estudiantes de ambos sexos tienen el mismo acceso y las mismas tareas que realizar cuando utilizan las plataformas de enseñanza virtual. Considerando de la literatura existente sobre el tema, creemos que es necesario continuar el trabajo en esta línea.

Este estudio posee tres importantes limitaciones que orientan los futuros trabajos relacionados. Primero, para validad los resultados es necesario una muestra mayor de individuos. Segundo, el tipo de muestreo voluntario y dentro de una única organización es una limitación para la generalización de los hallazgos. $Y$ tercero y último, el estudio es sólo un corte en el tiempo, sería aconsejable un estudio longitudinal para comparar las distintas etapas temporales de la adopción del e-learning.

\section{Referencias}

BATES, A. W. (2005). Technology, E-learning and Distance Education (2da ed.). Routledge.

CHANG, S., Tung, F. (2008). An empirical investigation of students' behavioural intentions to use the online learning course websites. British Journal of Educational Technology, 39 (I), 7I-83.

CHEN, Y., Chen, C., Lin, Y., Yeh, R. (2007). Predicting College Student' Use of E-Learning Systems: an Attempt to Extend Technology Acceptance Model. En Proceedings of PACIS 2007, Paper 121.

CHIN, W. W. (1998). The partial least squares approach for structural equation modeling. En: George A. Marcoulides (Ed.), Modern Methods for Business Research, Lawrence Erlbaum Associates.

CHIN, W.W. (2000). Frequently Asked Questions - Partial Least Squares \& PLS-Graph. http://discnt.cba.uh.edu/chin/plsfaq.htm [Accesado el 15 de Noviembre de 2009].

DAVIS, F.D. (1989). Perceived usefulness, perceived ease of use, and user acceptance of information technologies. MIS Quarterly, I3(3), 319-340.
FORNELL, C., Larcker, D.F. (I98I). Evaluating structural equation models with unobservable variables and measurement error. Journal of Marketing Research, 18, 3950.

GRANDON, E.E, Alshare, K., Kwun, O. (2005). Factors influencing student intention to adopt online classes: $A$ cross-cultural study. Consortium for Computing Sciences in Colleges, http://delivery.acm.org/10.1 | 45 / $050000 / \mid$ 047853/p46-grandon.pdf?key | $=\mid 047853 \&$ key2= 445384452 | \&coll=GUIDE\&dI=GUIDE\&CFID $=55770397 \%$

CFTOKEN=31674932 [Accesado el 2 de Octubre de 2009].

GUNASEKARAN, A., Mcneil, R. D., Shaul, D. (2002). Elearning: research and applications. Industrial and Comercial Training, 34(2), 44-53.

HADJERROUIT, S. (2006). Creating Web-based learning systems: an evolutionary development methodology. En Proceedings of the 2006 Informing Science and IT Education Joint Conference, Saldford, UK, I I9-I 44.

HALAWI, L., McCarthy, R. (2008). Measuring students perceptions of blackboard using the technology acceptance model: a PLS approach. Issues in Information Systems, 9(2), 95- 102.

HARRIS, M., Yanosky, R., Zastrocky, M. (2003). Supplemental Beats Remote in Higher-Education ELearning. Gartner Research Document, ID Number: COM20-0839.

HAYASHI, A., Chen, C., Ryan, T., Wu, J. (2004). The role of social presence and moderating role of computer selfefficacy in predicting the continuance usage of e-learning systems. Journal of Information Systems Education, I5(2), I39154.

KEIL, M., Tan, B. C. Y., Wei, K. K., Saarinen, T., Tuunainen, V., Wassenaar, A. (2000). A Cross-Cultural Study on Escalation of Commitment Behavior in Software Projects. MIS Quarterly, 24(2), 299-325.

KIM, J., Forsythe, S. (2008). Adoption of Virtual Try-on technology for online apparel shopping. Journal of Interactive Marketing, 22(2), 45-59. 
LEE, J.-S., Cho, H., Gay, G., Davidson, B., Ingraffea, A. (2003). Technology Acceptance and Social Networking in Distance Learning. Educational Technology \& Society, 6(2), 50-61.

LIU, S., Liao, H., Pratt, J.A. (2009). Impact of media richness and flow on e-learning technology acceptance. Computers \& Education, 52(3), 599-607.

MUÑOZ, M. (2005). Feasibility of e-learning at Bio-Bio University. Theoria, I4(2), 83-91.

NGAI, E. W. T., Poon, J. K. L., Chan, Y. H. C. (2007). Empirical examination of the adoption on WebCT using TAM. Computers \& Education, 48, 250-267.

O'NEILL, K., Singh, G., O'Donoghue, J. (2004). Implementing eLearning Programmes for Higher Education: A Review of the Literature. Journal of Information Technology Education, 3, 313-323.

OECD (2005). E-learning in Tertiary Education: Where Do we Stand? OECD, Paris.

ONG, C.S., Lai, J.Y. (2006). Gender differences in perceptions and relationships among dominants of elearning acceptance. Computers in Human Behaviour, 22 (5), 816-829.

ONG, C.S., Lai, J.Y., Wang, Y.S. (2004). Factors affecting engineers' acceptance of asynchronous e-learning systems in high-tech companies. Information \& Management, 4I (6), 795-804.

PARK, N., Lee, K. M., Cheong, P. H. (2007). University instructors' acceptance of electronic courseware: An application of the technology acceptance model. Journal of Computer-Mediated Communication, I3(I), artículo 9.

PARK, S. Y. (2009). An Analysis of the Technology Acceptance Model in Understanding University Students' Behavioral Intention to Use e-Learning. Educational Technology \& Society, 12(3), 150-162.

RAZA, A., Murad, H. S. (2008). Knowledge democracy and the implications to information access. Multicultural Education \& Technology Journal, 2 (I), 37-46.
RINGLE, C. M., Wende, S., Will, A. (2005). SmartPLS 2.0 (M3) beta, Hamburg: http://www.smartpls.de.

ROCA, J.C, Gagné, M. (2008). Understanding e-learning continuance intention in the workplace: A selfdetermination theory perspective. Computers in Human Behavior, 24(4), I585-1604.

ROCA, J.C., Chiu, C-M, Martínez, F.J. (2006). Understanding e-learning continuance intention: An extension of the Technology Acceptance Model. International Journal of Human-Computer Studies, 64(8), 683696

SAADÉ, R. G., Nebebe, F., Tan, W. (2007). Viability of the technology acceptance model in multimedia learning environments: Comparative study. Interdisciplinary Journal of Knowledge and Learning Objects, 37, 175- 184.

SAADE, R., Bahli, B. (2005). The impact of cognitive absorption on perceived usefulness and perceived ease of use in on-line learning: an extension of the technology acceptance model. Information Management, 42, 317-327.

SANCHEZ-FRANCO, M. J., Villarejo-Ramos, A. F., Rondan-Cataluña, F. J. (2006). Male and female professors. A theoretical analysis regarding the web acceptance and use. En Proceedings of the fifth European marketing trends congress, Venecia, I-I0.

TENENHAUS, M., Vinzi, V.E., Chatelin, Y.-M., Lauro, C. (2005). PLS path modeling. Computational Statistics and Data Analysis, 48, 159-205.

VAN RAAlJ, E.M., Schepers, J.J.L. (2008). The Acceptance and Use of a Virtual Learning Environment in China. Computers and Education, 50(3), 838-852.

VENKATESH, V., Bala, H. (2008). Technology Acceptance Model 3 and a Research Agenda on Interventions. Decision Sciences, 39(2), 273-3I5.

VENKATESH, V., Morris, M.G. (2000). Why don't men ever stop to ask for directions? Gender, social influence, and their role in technology acceptance and usage behavior. MIS Quarterly, 24(I), II5-139.

WALBY, S. (1990). Theorizing Patriarchy. Blackwell Publishers Ltd, Oxford.

ISSN: 07I 8-2724. (http://www.jotmi.org) 
YUEN, A.H.K., Ma, W.W.K. (2008). Exploring teacher acceptance of e-learning technology. Asia-Pacific Journal of Teacher Education, 36(3), 229-243.

ZHANG, D., Nunamaker, J.F. (2003). Powering e-learning in the new millennium: an overview of e-learning and enabling technology. Information Systems Frontiers, 5(2), 207-

218.

ZHANG, S., Zhao, J., Tan, W (2008). Extending TAM for Online Learning Systems: An Intrinsic Motivation Perspective. Tsinghua Science \& Technology, I3(3), 312-317. 